\title{
Anisotropic FCC Metal Nanostructures by Kinetically Controlled Synthesis
}

\author{
Jinguo Wang*, Yujie Xiong ${ }^{+}$, Byungkwon $\mathrm{Lim}^{+}$, Younan $\mathrm{Xia}^{+}$and M. J. Kim* \\ *Materials Science and Engineering, University of Texas at Dallas, RL10, Richardson, TX 75083 \\ ${ }^{+}$Department of Biomedical Engineering, Washington University, St. Louis, Missouri 63130
}

\begin{abstract}
Shape control of nanostructures has received considerable attention because the properties of nanostructures depend on their size, shape, composition, crystallinity, and structure. For facecentered cubic $(f c c)$ metals, there is no intrinsic driving force for the growth of anisotropic structures when the seeds are surrounded by an isotropic medium. Thermodynamically, fcc metal atoms are expected to nucleate and grow into cuboctahedrons (with a nearly spherical shape) enclosed by a mix of $\{111\}$ and $\{100\}$ facets to minimize the total surface energy. An $f c c$ metal can only be forced to grow into anisotropic nanostructures through kinetic control, although cubic symmetry is broken in this process. This paper describes a simple and versatile method for growing highly anisotropic nanostructures of fcc metals as well as HRTEM characterization of single crystal anisotropic fcc metal nanostructures.
\end{abstract}

Anisotropic fcc metal nanostructures were synthesized by a solution-phase method [1]. Structure characterization was performed in a JEOL 2100F field emission transmission electron microscope equipped with a Gatan Enfina 1000 spectrometer and EDAX energy dispersive x-ray spectrometer. Under Scherzer defocus, the JEOL $2100 \mathrm{~F}$ with a UHR pole piece has a point resolution of $1.9 \AA$. For fcc metals (such as $\mathrm{Pd}, \mathrm{Ag}$ ) viewed along $<100>$ or $<111>$ orientation, some of the interplanar distances are less than $1.9 \AA$. In order to resolve this, HRTEM images need to be taken at Lichte defocus which maximizes the information limit of the microscope to $1.0 \AA$ [2].

Figure 1 shows Ag particles nucleating and growing into cuboctahedrons (with a nearly spherical shape) enclosed by a mix of $\{111\}$ and $\{100\}$ facets that minimize the total surface area under normal condition. Figure 2 shows different shapes of Pd nanostructures under kinetically controlled synthesis; (A) a nanobar with an aspect ratio of 1 recorded along [010], (B) a nanobar with an aspect ratio of 2 recorded along [010], (C) a nanorod recorded along [1̄ㅣ, and (D) a nanorod recorded along [010]. Figure 3 shows the aging effect of Au nanoparticles and Pd nanobars (a) as synthesized and (b) 2 weeks later (Pd nano particles). The syntheses were performed with a molar ratio of $\mathrm{KBr}$ to $\mathrm{HAuCl} 4$ (or $\mathrm{Na} 2 \mathrm{PtCl}$ ) at 30 , in the presence of $75 \mathrm{mM} \mathrm{PVP}$, and in an 11-mL mixture of EG $(72.7 \%)$ and water at $140{ }^{\circ} \mathrm{C}$.

In summary, the anisotropic growth of fcc nanostructures can be achieved by varying the type and concentration of reducing agent, as well as by adjusting the reaction temperature. As expected for a kinetically controlled product, the anisotropic nanostructure evolved into the thermodynamically favored shape during an aging process [3].

[1] Yujie Xiong et al., J. Am. Chem. Soc., 129 (12) (2007), 3665 -3675.

[2] David. J. Smith, Ultramicroscopy 108 (2008) 159-166.

[3] This work was supported by a grant (code \#:08K1501-01210) from 'Center for Nanostructured Materials Technology' under ' $21^{\text {st }}$ Century Frontier R\&D Programs' of the Ministry of Education, Science and Technology Korea. 

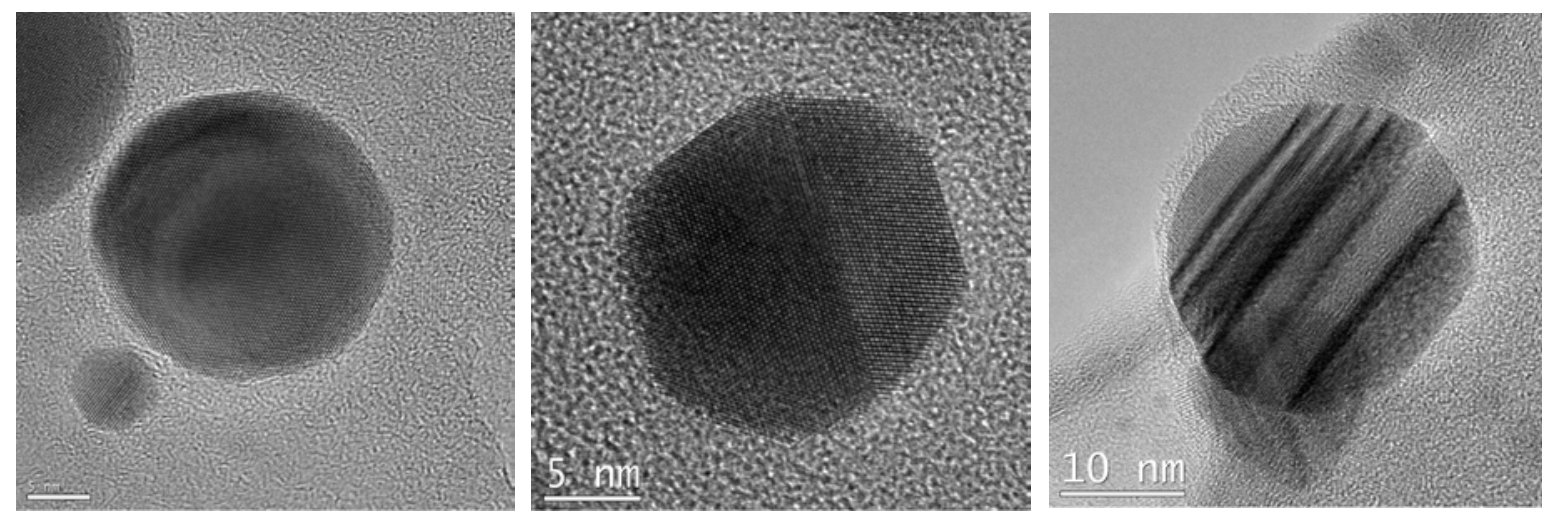

Figure 1. Ag particles nucleate and grow into cuboctahedrons (with a nearly spherical shape) enclosed by a mix of $\{111\}$ and $\{100\}$ facets in order to minimize the total surface area under normal condition.
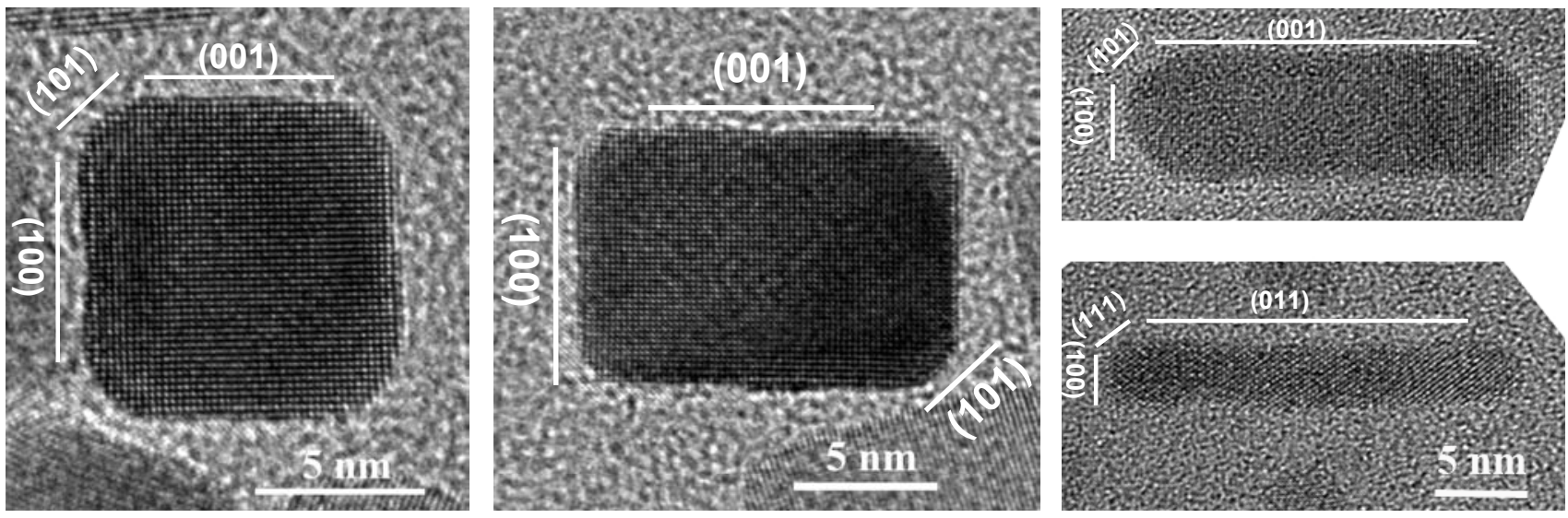

Figure 2. Different shapes of Pd nanostructures under kinetically controlled synthesis. (a) nanocube with an aspect ratio of $\sim 1$ under $100^{\circ} \mathrm{C}, 0 \%$ ethylene glycol, (b) nanobar with an aspect ratio of 2 , under $100^{\circ} \mathrm{C}, 9.1 \%$ ethylene glycol, and (c) nanorods under $120^{\circ} \mathrm{C}, 72.7 \%$ ethylene glycol.
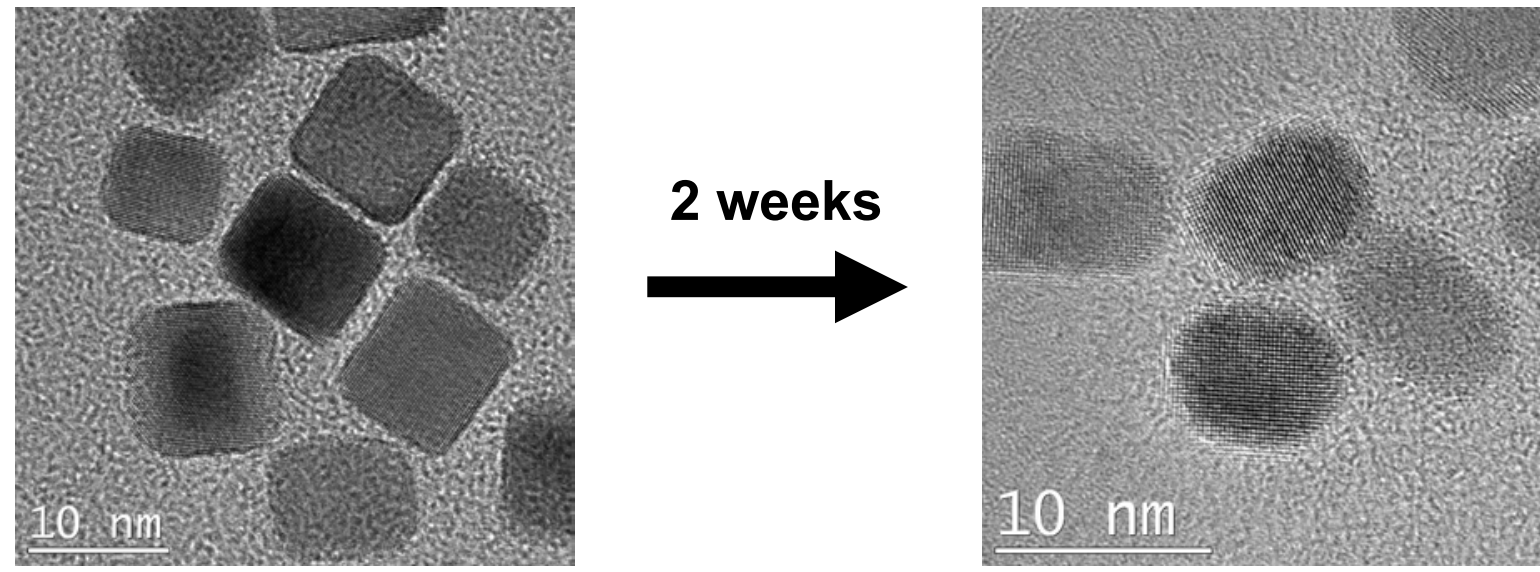

Figure 3. Aging effect of Pd nanoparticles: (a) as synthesized and (b) 2 weeks later. The syntheses were performed with a molar ratio of $\mathrm{KBr}$ to $\mathrm{Na}_{2} \mathrm{PtCl}_{6}$ at 30 , in the presence of $75 \mathrm{mM} \mathrm{PVP}$, and in an 11-mL mixture of EG (72.7\%) and water at $140{ }^{\circ} \mathrm{C}$. 